\title{
THOMAS MEHLHAUSEN
}

Poczdam

\section{Die Osterweiterung der Europäischen Union. Die anomischen Konsequenzen der deutschen Wiedervereinigung}

Die neueste Geschichte der deutsch-polnischen Beziehungen steht im Zeichen der europäischen Integration. Nicht zuletzt vor dem Hintergrund des gemeinsam verfolgten Ziels eines polnischen Beitritts in die Europäische Union (EU) charakterisierte der damalige polnische Außenminister Krzysztof Skubiszewski im Jahr 1993 das Verhältnis zwischen Deutschland und Polen als eine „Interessengemeinschaft“. Deutschland zählte nicht nur zu den größten Befürwortern einer Osterweiterung, sondern insbesondere eines Beitritts Polens.

Im folgenden Beitrag wird argumentiert, dass es paradoxerweise gerade die Wiedervereinigung Deutschlands war, die zur anfänglichen Zögerlichkeit in der EU-Erweiterungspolitik führte. Solange die Ratifizierung des Maastrichter Vertrags nicht als sicher galt, konnten die Erweiterungsgegner innerhalb der EG diese für sie kostspielige Entscheidung mit dem Hinweis darauf aufschieben, dass zunächst das , deutsche Problem' institutionell bewältigt werden müsse: Immerhin bedeutete die Wiedervereinigung für Deutschland einen erheblichen Machtzuwachs, der sich durch eine Osterweiterung zusätzlich erhöht hätte, da Deutschland als ihr größter Profiteur galt. Erst nachdem den erweiterungsskeptischen Mitgliedstaaten infolge der Ratifizierungen des Maastrichter Vertrags in Dänemark und Großbritannien kein legitimes Gegenargument mehr zur Verfügung stand, gelang es den Erweiterungsbefürwortern sich durchzusetzen, indem sie den Widerspruch zwischen der liberalen Identität der Gemeinschaft und der Verweigerung einer Beitrittsperspektive für die mittelosteuropäischen Transformationsstaaten offen legten. Zur Vertiefung dieses Arguments wird ein handlungstheoretisches Analyseinstrumentarium präsentiert, das die Theorie des rhetorischen Handelns (Frank Schimmelfennig) durch eine Integration des soziologischen Konzepts der Anomie weiterentwickelt. Dadurch wird ein Anschluss an die theoretische Debatte in den Internationalen Beziehungen zwischen Rationalismus und Konstruktivismus ermöglicht.

Im ersten Abschnitt soll die theoretische Debatte zur Osterweiterung der EU rekapituliert werden, um die verbleibenden Erklärungsdesiderate zu identifizieren. Nachfolgend wird auf empirischer Ebene demonstriert, dass die Erkenntnisse aus der Debatte über die Konsequenzen der deutschen Wiedervereinigung für die Einigung auf den Maastrichter Vertrag die inkonsistente Erweiterungspolitik besser verstehen lassen. Schließlich werden erst allgemeine Erwartungen bezüglich des Verhaltens so genannter ,schwach sozialisierter Akteure' (Schimmelfennig) formuliert, wenn deren materielle Partikularinteressen im Widerspruch zu den vorherrschenden Gemeinschaftsnormen stehen, um so demonstrieren zu können, dass - den Erwartungen entsprechend 
- sowohl ökonomische als auch normative Motive die inkonsistente Erweiterungspolitik erklären.

\section{Die theoretische Debatte über die Osterweiterung der Europäischen Union}

In der Erweiterungsliteratur besteht weitgehend Einigkeit darin, dass sich die EG in den ersten Jahren nach dem Fall des Eisernen Vorhangs zögerlich (vgl. Torreblanca 2001: 1) und wenig großzügig zeigte (vgl. Grabbe, Hughes 1998: 29; Saryusz-Wolski 1994: 21) ${ }^{1}$. Dies lässt sich anhand von drei Beobachtungen illustrieren. Erstens gab sie den mittel- und osteuropäischen Staaten trotz erster Transformationserfolge keine Beitritts-perspektive. Wie der polnische Unterhändler in den Verhandlungen zu den Assoziierungsverträgen, Jarosław Mulewicz (1992: 30), dem Sejm berichtete, konnte nur auf Drängen Ungarns erreicht werden, dass darin eine Erweiterung allenfalls als Ziel der Beitrittsaspiranten Erwähnung fand. Ein explizites Ziel der EG war es nicht. Zweitens schien die finanzielle Unterstützung angesi chts des enormen Bedarfs eher dürftig. John O'Brennan (2006: 15-17) errechnete, dass der Umfang nicht einmal ein Zehntel des Marshall-Plans umfasste. Drittens wirkten sich die Handelsvereinbarungen in den Assoziierungsverträge sogar nachteilig auf die Handelsbilanzen aus, da die EG eine Liberalisierung gerade der Sektoren verweigerte, in denen Polen und die anderen Anwärter konkurrenzfähig waren (vgl. Sedelmeier 2005: 54). Dies betraf Agrar- und Textilprodukte, Kohle und Stahl.

Vor diesem Hintergrund überraschte die Erweiterungsentscheidung im Juni 1993, als sich der Europäische Rat in Kopenhagen erstmals die Aufnahme der mittelosteuropäischen Staaten ausdrücklich als eigenes Ziel setzte und in Form der Kopenhagener Beitrittskriterien die Bedingungen dafür explizierte. Dies stellt theoretische Erklärungsversuche vor drei wichtige Fragen: Erstens, warum legte kein Mitgliedstaat ein Veto ein? Staaten wie Portugal und Spanien konnten sich sicherheitspolitisch und ökonomisch kaum einen Gewinn versprechen. Zweitens, warum wurde von der EG kein alternatives institutionelles Arrangement unterhalb des Niveaus einer Vollmitgliedschaft präferiert? Und schließlich, drittens, warum fiel die Erweiterungsentscheidung gerade im Juni 1993, und nicht früher oder später (Timing)?

Dieser sonderbare Verlauf der EU-Osterweiterung löste eine intensive theoretische Debatte aus (siehe Schimmelfennig, Sedelmeier 2005a). Realistische Zugänge betrachten relative Machtgewinne und insbesondere Sicherheitsschocks als die entscheidenden Erklärungsvariablen (Skalnes 2005; Vobruba 2004, 2005; Smith 2004). Doch einer Stabilisierungspolitik entsprach die Phase bis 1993 nicht, da die EG durch die Assoziierungsverträge keineswegs den potenziellen Konflikte in Mittelosteuropa präventiv entgegenwirkte: Zum einen wurden den Reformeliten durch die Verweigerung der Beitrittsperspektive wichtige Anreize zur Systemtransformation entzogen, denen in der Li-

1 Im Folgenden werde ich die Bezeichnung „Europäische Union“ verwenden, es sei denn ich beziehe mich explizit auf den Zeitraum vor 1993. Bis zum Inkrafttreten des Maastrichter Vertrags am 1.11.1993 lautete die offizielle Bezeichnung Europäische Gemeinschaft, danach erst Europäische Union. 
teratur zur Konditionalisierung erhebliches Demokratisierungspotenzial zugesprochen wird (vgl. Risse, Börzel 2009; Schimmelfennig, Sedelmeier 2005b; Vachudova 2005). Zum anderen drohten die für die mittelosteuropäischen Beitrittsaspiranten ungünstigen Handelsvereinbarungen und die geringe finanzielle Unterstützung die Staaten in einer frühen Transformationsphase zu destabilisieren, da dadurch eine steigende Arbeitslosigkeit, Wirtschaftsrezession und sinkendes Vertrauen der Bevölkerungen in den Transformationserfolg zu befürchten waren.

Liberale Ansätze (Moravcsik, Vachudova 2002; siehe auch Moravcsik 1998) können aus einer handelspolitischen Perspektive die Assoziierungsverträge gut erklären. Sowohl die Einteilung der Mitgliedstaaten in Beitrittsbefürworter und -Gegner als auch die konkreten Verhandlungsergebnisse in den Assozi ierungsverträgen - insbesondere die Verweigerung einer Handelsliberalisierung in einigen ,sensiblen' Sektoren - entsprachen den Erwartungen des liberalen Intergouvernementalismus (vgl. Schimmelfennig 1999). Doch die Abkehr von dieser pareto-optimalen Konstellation bleibt rätselhaft. Einige Autoren versuchen die Erweiterungsentscheidung durch Berücksichtigung künftiger Gewinne und Kosten zu erklären. Auf der einen Seite wird argumentiert, dass die künftigen Kosten durch Übergangs fristen abgemildert werden (Plümper, Schneider 2007; Schneider 2009); auf der anderen Seite werden zusätzliche Gewinne in einem größeren Einflusspotenzial für Lobbygruppen aus den alten Mitgliedstaaten in einer erweiterten EU identifiziert (Brou, Ruta 2004; Kemmerling 2004). Aufgrund des Folk-Theorems können jedoch weder mögliche künftige Gewinne noch abge milderte Kosten die Erweiterungsentscheidung erklären, ohne zu zeigen, dass es für die potenziellen Erweiterungsverlierer in der Gegenwart nennenswerte Vorteile im Vergleich zum Status quo gibt ${ }^{2}$.

Konstruktivistische Zugänge rekurrieren auf die liberale Iden tität der EU. Formal legt der Artikel 49 des Vertrags über die Eu ropäische Union Folgendes fest: ,Jeder europäische Staat, der die in Artikel 2 genannten Werte [Achtung der Menschenwürde, Freiheit, Demokratie, Gleichheit, Rechtsstaatlichkeit, Wahrung der Men schenrechte einschließlich der Recht der Personen, die Minderheiten angehören sowie Pluralismus, Nichtdiskriminierung, Toleranz, Gerechtigkeit, Solidarität und Gleichheit von Frauen und Männern, T. M.] achtet und sich für ihre Förderung einsetzt, kann beantragen, Mitglied der Union zu werden“. Fierke und Wiener (1999) sehen insbesondere in der Schlussakte von Helsinki von 1975 eine Manifestation der liberalen Rhetorik des Kalten Kriegs und interpretieren dies als explizites künftiges Beitrittsversprechen. Um nicht im Widerspruch zu jener Verpflichtung zu stehen und dadurch die eigene Identität zu unterminieren, sei die EG später gezwungen gewesen, den beitrittswilligen Staaten eine Mitglied schaftsperspektive zu gewähren. Während die Erweiterungsentscheidung aus dieser Sicht als eine Frage der Glaubwürdigkeit von Mitgliedern einer liberalen Gemeinschaft nur folgerichtig erscheint, bleibt wiederum die wenig großzügige Assoziierungs phase widersprüchlich (vgl. Schimmelfennig 2003: 156).

Eine Reihe von Ansätzen wählt einen synthetischen Zugang, der weder rein eigennutzorientierte noch ausschließlich nor mengeleitete Akteure unterstellt (Torreblanca

${ }^{2}$ Dem Folk-Theorem zufolge muss der Diskontfaktor $\delta \geq 1$ betragen, d.h. der Zukunftsnutzen wird nicht stärker als der gegenwärtige Nutzen gewichtet. 
2001; Sedelmeier 2002, 2005). In Bezug auf die Ratsentscheidung schlägt Frank Schimmelfennig $(2001,2003)$ die Theorie des rhetorischen Handelns vor, der zufolge schwach sozialisierte Akteure zwar ihre materiellen Interessen zu realisieren versuchen - wie von Vertretern rationalistischer Theorien unterstellt - doch gleichzeitig als verlässliche Gemeinschaftsmitglieder wahrgenommen werden wollen - wie Sozialkonstruktivisten annehmen. Schim melfennig argumentiert nun, dass die Erweiterungsbefürworter ihre Kontrahenten sukzessive durch Bezugnahme auf die o.g. Offenheit der Europäischen Union gegenüber europäischen und liberal verfassten Staaten in eine rhetorische Falle drängten. Um angesichts der früheren Bekenntnisse zu den liberalen Wer ten der EG nicht als unglaubwürdige Gemeinschaftsmitglieder wahrgenommen zu werden, waren sie schließlich trotz erwarteter Kosten bereit - und damit nicht aus Überzeugung sondern Opportunismus - der Osterweiterung zuzustimmen.

Trotz der plausiblen Argumentation Schimmelfennigs, die die spezifische Phasenabfolge in der Erweiterungspolitik verstehen lässt, bleibt das Timing weiterhin ungeklärt. Warum waren die Erweiterungsbefürworter nicht früher in der Lage, ihre Kontrahenten zur schweigenden Zustimmung zu zwingen? Eine Antwort liefert die Analyse der institutionellen Konsequenzen der deutschen Wiedervereinigung. Während die Bedeutung für die Vertiefung, also die Einigung auf den Maastrichter Vertrag, in der Literatur breit diskutiert wurde, blieb eine Betrachtung der Folgen für die Osterweiterung aus. Dabei kann gezeigt werden, dass die Unsicherheit innerhalb der EG infolge der Wiedervereinigung die Zögerlichkeit der anfänglichen Assoziierungsphase auslöste.

\section{Deutsche Wiedervereinigung und europäische Integration}

Seit Beginn der europäischen Integration herrschte die Überzeugung vor, dass die Delegation nationaler Souveränität nur dann legitim ist, wenn den Mitgliedstaaten ein gleichberechtigter Zugang zum gemeinsamen Entscheidungsprozess gewährt wird. Diesen Gedanken formulierte Winston Churchill frühzeitig in seiner viel beachteten Rede im September 1946: „Small nations will count as much as large ones and gain their honor by their contribution to the common cause". (Churchill 1946) Ähnlich äußerte sich auch der damalige französische Außenminister Robert Schuman:

"Die Schaffung dieser mächtigen Produktionsgemein schaft, die allen Ländern offensteht, die daran teilnehmen wollen, mit dem Zweck, allen Ländern, die sie umfasst, die notwendigen Grundstoffe für ihre industrielle Produktion zu gleichen Bedingungen zu liefern, wird die realen Fundamente zu ihrer wirtschaftlichen Vereinigung legen. [...] Die gemeinsame Hohe Behörde, die mit der Funktion der ganzen Verwaltung betraut ist, wird sich aus unabhängigen Persönlichkeiten zusammensetzen, die auf paritätischer Grundlage von den Regierungen ernannt werden“. (Schuman 1950, eigene Hervorhebung).

Dabei konkurriert das Prinzip der Souveränität mit dem demokratischen Prinzip. Ersteres erfordert eine Egalität der Mitgliedstaaten und spiegelt sich in der Einstimmigkeitsregel wider; letzteres impliziert eine Egalität der Bürger und findet seine explizite Entsprechung in der direkten Proportionalität bei demokratischen Wahlen. Es ist das 
Dilemma dieser dualen Egalität, dass aufgrund der unterschiedlichen Bevölkerungsgrößen der Mitgliedstaaten die Gewährleistung der einen Egalität mit der der anderen nicht vereinbar ist. Diese duale Egalität lässt sich im Prin zip der degressiven Proportionalität in den Abstimmungsverfahren bzw. der Repräsentation der Mitgliedstaaten in den EU-Institutionen erkennen, in denen von der Einstimmigkeits- bzw. Gleichverteilungsregel abgerückt wurde. Es besagt, dass der Quotient aus Bevölkerung und Sitzverteilung (bzw. Stimmenverteilung) bei sinkender Bevölkerung fallen muss, d..h. ein bevölkerungsreicher Mitgliedstaat entsendet zwar mehr Vertreter, doch diese vertreten gleichzeitig mehr Unionsbürger als solche von kleinen Mitglied staaten. Während die Proportionalität verhindert, dass die Stimmen der Bürger kleiner Staaten deutlich stärker wiegen als die von großen, gewährleistet die Degressivität, dass der Einfluss kleiner Staaten infolge der Egalität der Bürger nicht in die Bedeutungslosigkeit versinkt. Zwar wurde dieses Prinzip erst 2007 vertraglich explizit in Bezug auf die Zusammensetzung des Europäischen Parlaments genannt, doch es lässt sich ebenso bei der Stimmengewichtung im Rat und in der Zusammensetzung der Kommission bis 2004 erkennen. Dabei schließt jene partizipative Egalität die Dominanz einer oder weniger Staaten über die anderen aus. Das prominenteste Beispiel ist der Luxemburger Kompromiss, wonach ein Mitgliedstaat bei einer drohenden Überstimmung gemäß qualifizierter Mehrheit den Abstimmungsgegenstand zu einem Objekt vitaler nationaler Interessen erheben und die Entscheidung faktisch verhindern kann. Aber auch die Fokussierung auf Sperrminoritäten bei der Reformierung des Abstimmungssystems im Ministerrat während der Verhandlungen zum Europäischen Verfassungsvertrag im Jahr 2004 verdeutlicht die Sensibilität mancher Staaten gegenüber potenziell allmächtigen Mehrheitskoalitionen in diesem Gremium (siehe Mehlhausen 2009).

Die deutsche Wiedervereinigung bedrohte jene partizipato rische Egalität aufgrund eines erheblichen Machtanstiegs zugunsten Deutschlands. Die europäische Integration begann vor dem Hintergrund zweier von Deutschland begonnener, katastrophaler Kriege in der ersten Hälfte des 20. Jahrhunderts. Insofern beunruhigte die Machtsteigerung des ehemaligen Aggressors in dreifacher Hinsicht: Erstens wuchs nicht nur die Bevölkerung des bisher bevölkerungsreichsten Mitgliedstaats (um 25,1 Prozent) sondern auch sein Staatsgebiet (um 43,7 Prozent). Zweitens konnte Deutschland aufgrund der gestiegenen Bevölke rungsgröße einen Anspruch auf einen höheren institutionellen Einfluss geltend machen. Die bereits erwähnte aus dem demokratischen Prinzip abgeleitete Egalität der Bürger verlangt eine entsprechende Repräsentation des größeren Anteils Deutschlands an der Unionsbevölkerung. Ein institutioneller Machtanstieg wiegt im Falle Deutschlands umso stärker, da es als bereits größte Volkswirtschaft innerhalb der EU sein ökonomisches Potenzial im Verhandlungsprozess strategisch einzusetzen vermag, indem es mit Hilfe von Seitenzahlungen Kompromisse zu seinen Gunsten aushandelt. Drittens erhielt Deutschland durch die Zwei-plus-Vier-Verträge die volle Souveränität zurück. Der bereits doppelte Machtgewinn wurde somit durch den Wegfall der direkten Interventionsfähigkeit der Alliierten weiter gesteigert. Wenngleich sich alle führenden deutschen Politiker einer Kontinuität der Außenpolitik verpflichteten, besaß Deutschland nun zumindest das Potenzial, eine stärker unilateral ausgerichtete Außenpolitik zu betreiben. Die Bedenken in Frankreich und Großbritannien waren groß. Elizabeth Pond schrieb dazu: 
"The French feared German might unrestrained by the EC, but they also feared German might magnified by the EC." (sic!) (Pond 1993: 211) Eine ähnliche Erwartung formulierten William Paterson und Simon Bulmer (1987: 232): „A reunified Germany would not inevitably wish to remain an EC member. Even if it did, a new state of such size and power might fatally unbalance the Community."

Der Vertrag von Maastricht enthielt die passenden institutionellen Lösungen für die dreifache Herausforderung. Die Rückgewinnung der vollen Souveränität wurde durch eine zunehmende Vertiefung und insbesondere die Schaffung der zweiten Säule, der Gemeinsamen Außen- und Sicherheitspolitik, kompensiert. Dem gestiegenen potenziellen Repräsentationsanspruch Deutschlands entsprach man nur bedingt durch eine allenfalls geringe institutionelle Anpassung, da keine Veränderungen an der Zusammensetzung der Europäischen Kommission sowie der Stimmenverteilung im Ministerrat vorgenommen wurden. Deutschland wurden lediglich 18 zusätzliche Sitze im Europä ischen Parlament gewährt. Letztere werden allerdings meistens als Verhandlungsmasse eingesetzt (Moberg 2002: 280) und deuten auf nur kleinere Zugeständnisse hin. Schließlich kann die Einigung auf die Wirtschafts- und Währungsunion, durch die die starke Deutsche Mark in die Gemeinschaftswährung Euro überführt wurde, auch als eine Reaktion auf den Machtanstieg Deutschlands gewertet werden (Garrett 1993: 105; Milward, Sorensen 1993: 29; Pond 1993: 159). Dies erinnert an ein Grundmotiv der europäischen Einigung. Schon Anfang der 1950er Jahre hatte man eine strategisch wichtige Ressource eines Mitgliedstaates - damals Kohle und Stahl, nun die Währung - der Gemeinschaft zugänglich gemacht. Moravcsik (1998: 381) wendet ein, dass Deutschland bereits vor der Wiedervereinigung für einen gemeinsamen Binnenmarkt und die Einführung einer Gemeinschaftswährung plädierte. Doch angesichts der deutschen Wiedervereinigung wurde aus einer vormals ökonomischen Option nun eine politische Notwendigkeit.

Wenngleich diese institutionelle Lösung das Gleichgewicht innerhalb der Gemeinschaft wieder hergestellt hätte, galt die Ratifizierung der Einigung vom Dezember 1991 keineswegs als sicher. Während das Referendum zum Maastrichter Vertrag in Frankreich mit 51 Prozent zwar positiv aber denkbar knapp aus fiel, scheiterte das Referendum in Dänemark im Juni 1992. Erst nachdem das wiederholte Referendum am 18. Mai 1993 erfolgreich verlief und das britische House of Commons zwei Tage später ebenfalls ratifizierte, schien das Inkrafttreten des EU-Vertrags garantiert. Einen Monat später entschied sich der Europäische Rat in Kopenhagen für die Osterweiterung, indem er spezielle Kriterien die von den Anwärtern zu erfüllenden Beitrittsbedingungen explizierte. Doch in welchem kausalen Zusammenhang standen die vertikale und die horizontale Dimension der euro päischen Integration?

Ich argumentiere, dass die Ratifizierung des Maastrichter Vertrags deswegen zur notwendigen Bedingung für ein progressiveres Engagement in der Erweiterungspolitik wurde, da sich der ohnehin gegebene Machtzuwachs Deutschlands durch die Osterweiterung weiter erhöht hätte. (Ausführlicher dazu Mehl hausen 2009: 96-114) Schließlich galt Deutschland als größter Profiteur der Osterweiterung. Folglich konnten sich die Erweiterungsgegner bis 1993 erfolgreich durchsetzen, indem sie auf die Lösung des ,deutschen Problems' bestanden. Auf der Kopenhagener Regierungskonferenz im Juni 1993 fehlte ihnen jedoch ein legitimes Argument gegen eine Osterweiterung und so mussten sie einlenken. Im Folgenden soll diese Argumentation theoretisch vertieft werden. 


\section{Schwach sozialisierte Akteure in diffusen Entscheidungskontexten}

Die große Debatte in den Internationalen Beziehungen lässt sich auf die Frage fokussieren, welcher Verhaltenslogik Staaten folgen. Der Großteil der Literatur, vor allem der Realismus und der Liberalismus, unterstellt einen Nutzen maximierenden, egoistischen homo oeconomicus, der sich in seinem Handeln stets an seinen a priori definierten materiellen Interessen orientiert. Diesem Akteursverständnis stellen Sozialkonstruktivisten einen in seiner sozialen Umwelt angemessen handelnden homo sociologicus entgegen, der materielle Interessen verfolgen kann, aber nur wenn dies den in seinem Umfeld vorherrschenden sozialen Normen entspricht. Dabei wird unterstellt, dass sich die entscheidungsrelevanten Interessen aus der spezifischen Identität der Akteure ableiten und durch neue Ideen oder einen Bedeutungswandel bestehender Normen beeinflusst werden können. Im Folgenden soll Schimmelfennigs Konzeption eines schwach sozialisierten Akteurs übernommen werden, die diese einflussreiche Dichotomie von Rationalismus und Konstruktivismus (siehe March, Olsen 1989; Hall, Taylor 1996) durchbricht. Wie bereits erläutert, folgt ein so charakterisierter Akteur weder einer rein rationalistischen noch einer ausschließlich normengeleiteten Verhaltenslogik, sondern einer rhetorischen, d.h. er versucht seine materiellen Interessen umzusetzen und zugleich mit einer anerkannten Gemeinschaftsnorm zu rechtfertigen, um als glaubwürdiges Gemeinschaftsmitglied wahrgenommen zu werden. Sollte ihn ein anderer Akteur bei der Verfolgung seiner materiellen Interessen mit einer diesem Verhalten widersprechenden Gemeinschaftsnorm konfrontieren, so kann er Schimmelfennig zufolge prinzipiell drei Strategien verfolgen. Er kann entweder die Glaubwürdigkeit des ihn herausfordernden Akteurs, die Legitimität der Gemeinschaftsnorm oder die Anwendbarkeit der Gemeinschaftsnorm in dem konkreten Entscheidungskontext in Frage stellen (Schimmelfennig 2003: 208-225). Scheint keine dieser Gegenstrategien vielversprechend zu sein, wird er einlenken müssen.

Entscheidungskontexte werden im Folgenden als diffus be zeichnet, wenn Gemeinschaftsnormen und materielle Interessen divergierende Handlungen suggerieren. Dabei kann zwischen schwachen und starken Intensitäten differenziert werden. Wie in makroökonomischen Modellen üblich, können materielle Interessen einander neutralisieren. Dann ist ein Akteur indifferent gegenüber einer Entscheidung. Aber auch Normen können einander widersprechen und zu einer Anomie führen ${ }^{3}$. Eine Anomie herrscht vor, wenn fundamentale und vergleichbar anerkannte Gemeinschaftsnormen unterschiedliche Entscheidungen legitimieren.

3 Der Begriff ,Anomie' ist durch Emilie Durkheim populär geworden und fand später in der Soziologie größere Beachtung. Die Anwendung des Begriffs unterscheidet sich zwar in Bezug auf seine Ursache - bei Durkheim der schwindende Legitimationsglaube, hier die Konkurrenz zu anderen Normen mit konträren Handlungsimplikationen - doch entscheidend ist im Folgenden die Konsequenz der Anomie: In beiden Fällen ist ein sy stematisches Abweichen von einer vormals konstitutiven Norm zu beobachten. Ähnlich verhält es sich bei der Diffusion einer Anomie: Während hier nicht ein wachsendes Regel vertrauen, sondern die klar wahrnehmbare Dominanz einer Norm in einem spezifischen Entscheidungskontext die Ursache ist, herrscht in beiden Fällen nun wieder eine nor menkonforme Verhaltenskultur vor. Eine ähnliche Adaption des Konzepts findet sich bei Götze 2003. 
Diese Überlegungen führen zu der folgenden Matrix (Abbildung 1). Ich unterstelle hier, dass ein Akteur eine Entscheidung, die einer Gemeinschaftsnorm entspricht, aufgrund seiner materiellen Interessen abzulehnen gewillt ist. Im ersten Entscheidungskontext sind beide Intensitäten stark. Es ist abhängig von den o.g. Gegenstrategien, ob die schwach sozialisierten Akteure der Entscheidung schweigend zustimmen müssen oder sich dagegen erfolgreich wehren können. Im zweiten Entscheidungskontext ist angesichts der bestehenden Anomie und starker Interessen eine Ablehnung zu erwarten, da dieses egoistische Verhalten mit einer opportunen Gemeinschaftsnorm gerechtfertigt werden kann. Analog dazu werden die schwach sozialisierten Akteure bei Indifferenz und einer vorherrschenden Norm der Entscheidung zustimmen, da sie sich ohne bzw. bei geringen Kosten als glaubwürdige Gemeinschaftsmitglieder inszenieren können. Bei Anomie und Indifferenz ist erneut nicht per se erkennbar, wie sich der schwach sozialisierte Akteur entscheidet, da keine bzw. schwache Handlungsanreize existieren. In diesem Fall ist eine Anfälligkeit für exogene Faktoren wahrscheinlich. Mit diesen allgemeinen Erwartungen zum Verhalten schwach sozialisierter Akteure soll nun der Verlauf der Osterweiterung der EU untersucht werden.

\begin{tabular}{|c|c|c|}
\hline $\begin{array}{l}\text { Intensität des normativen } \\
\text { Kontexts } \\
\text { Intensität der materiellen Interessen }\end{array}$ & hoch (eine Norm dominiert) & niedrig (Anomie) \\
\hline Hoch & $\begin{array}{l}\text { DEK 1: Zustimmung oder } \\
\text { Ablehnung } \\
\text { rhetorisches Handeln oder Gegen- } \\
\text { strategien möglich }\end{array}$ & $\begin{array}{l}\text { DEK 2: Ablehnung } \\
\text { materielle Interessen dominieren }\end{array}$ \\
\hline niedrig (Indifferenz) & $\begin{array}{l}\text { DEK 3: Zustimmung } \\
\text { normenkonformes Verhalten }\end{array}$ & $\begin{array}{l}\text { DEK 4: Zustimmung oder } \\
\text { Ablehnung } \\
\text { hohe Sensibilität für exogene Einfl- } \\
\text { üsse }\end{array}$ \\
\hline
\end{tabular}

DEK $=$ diffuse Entscheidungskontexte

Abb. 1: Akteursverhalten bei divergierenden Handlungsimplikationen der materiellen Interessen und einer Gemeinschaftsnorm.

\section{Diffuse Entscheidungskontexte und die Osterweiterung der Europäischen Union}

Diffuse Entscheidungskontexte dominierten den Verlauf der Osterweiterung der Europäischen Union. Die ökonomischen Interessen definierten weitgehend die Einteilung in Beitrittsbefürworter und -gegner. Während von den 15 Mitgliedstaaten nur Deutschland, Österreich, Großbritannien, Dänemark, Schweden und Finnland von Anfang an für eine Erweiterung plädierten, nahmen die restlichen Staaten eine skeptische bis ablehnende Haltung ein (Schimmelfennig 2001: 50). Die materiellen Interessen der Erweiterungsgegner waren stark ausgeprägt in Bezug auf redistributive Güter, um die sie nach einer Erweiterung mit den neuen Mitgliedstaaten konkurrieren müssen - v.a. die nationalen Kontingente der Agrar- und Strukturfonds. Im Gegensatz dazu fielen die handelspolitischen Kosten einer Erweiterung für sie zwar größer als für z.B. Deutschland aus, da sie auf ähnliche Sektoren spezialisiert waren wie die beitrittswilligen Staa- 
ten, doch gemessen an der Außenhandelsbilanz dieser EG-Mitgliedstaaten schien die Konkurrenz aus Mittelosteuropa eher schwach.

Der normative Kontext war bis 1993 durch eine Anomie geprägt. Sowohl die Offenheit der Europäischen Union gegenüber europäischen und liberal verfassten Staaten - im Folgenden ,inklusive Norm“ genannt - als auch die oben erläuterte partizipative Egalität im gemeinsamen Entscheidungsprozess - nun als ,kohäsive Norm“ bezeichnet - können als konstitutive und allgemein anerkannte Gemeinschaftsnormen betrachtet werden. Bis 1993 war allerdings die Realisierung der inklusiven Norm nicht mit der der kohäsiven Norm vereinbar, da eine Osterweiterung das ,deutsche Problem' verschärft hätte. Die Anomie löste sich erst nach den erfolgreichen Ratifizierungen in Dänemark und Großbritannien auf, infolge derer das Inkrafttreten des Maastrichter Vertrags als gewiss galt.

Die Beobachtungen entsprechen dem zuvor vermuteten Ver haltensmuster schwach sozialisierter Akteure in diffusen Entscheidungskontexten (Abbildung 2). Während die Gegner die Erweiterungsentscheidung erfolgreich verzögern konnten (Verhalten 1), bis die deutsche Wiedervereinigung innerhalb der EG institutionell bewältigt wurde und erst nach Auflösung der Anomie aufgrund des rhetorischen Handelns der Erweiterungsbefürworter einlenken mussten (Verhalten 3), lehnten sie handelspolitische Konzessionen im Schatten der Anomie ab (Verhalten 2) und lenkten erst 1993 ein (Verhalten 4).

Insbesondere das Verhalten im ersten und vierten Entscheidungskontext (Abbildung 1), in denen die konkurrierenden Handlungsanreize von ähnlicher Intensität sind, scheint aufgrund der theoretischen Ergebnisoffenheit erklärungsbedürf tig. In Bezug auf das Verhalten bei geringen materiellen Kosten und Anomie (Verhalten 2 in Abbildung 2) kann auf die Arbeit von Dimitris Papadimitriou (2002: 53.ff.) rekurriert werden, der mit Rückgriff auf den Ansatz der ,nested games““ (Tsebelis 1988) überzeugend demonstriert, dass die zeitgleichen GATT-Verhandlungen Frankreich keine handelspolitischen Zugeständnisse in den Assoziierungsverträgen erlaubten. Eine harte Verhandlungsstrategie wäre bei einer gleichzeitig kompromissbereiten Haltung gegenüber den mittelosteuropäischen Staaten - insbesondere von den USA - als inkonsistentes Verhalten kritisiert worden. Dieser exogene Faktor scheint den Protektionismus der EG in den Assoziierungsverträgen gut zu erklären. Im Gegensatz dazu lässt sich das Akteursverhalten bei starken materiellen und normativen Handlungsanreizen (Verhalten 3, Abbildung 2) - wie oben dargelegt - überzeugend mit Hilfe des Ansatzes von Schimmelfennig erklären: Mangels erfolgsversprechender Gegenstrategien waren die Erweiterungsgegner gezwungen einzulenken.

\section{Schlussfolgerungen}

Die deutsche Wiedervereinigung hatte nicht nur Konsequenzen für die vertikale sondern auch für die horizontale Dimension der europäischen Integration. Zwar wurde sie in der theoretischen Debatte zum Maastricht-Vertrag von einer Reihe von Autoren als ausschlaggebende Kontextbedingung identifiziert, doch in den Erklärungsansätzen zur Osterweiterung fand der erhebliche Machtzuwachs Deutschlands keine Berück- 
sichtigung. Dabei war es die deutsche Wiedervereinigung, die den Erweiterungsgegnern innerhalb der Europäischen Gemeinschaft ein legitimes Argument gegen eine progressivere Erweiterungspolitik bis 1993 lieferte. Erst nachdem das Inkrafttreten des neuen Vertrags aufgrund der Ratifizierungen in Dänemark und Großbritannien als sicher galt, mussten die Erweiterungsgegner einer für sie mit erheblichen Kosten verbundenen Osterweiterung zustimmen, um nicht als unglaubwürdige Mitglieder einer liberalen Gemeinschaft diskreditiert zu werden.

Auf einer theoretischen Ebene wurde gezeigt, dass weder rein ökonomisch-rationalistische noch soziologisch-konstruktivistische Ansätze den Verlauf der Osterweiterung erklären können. Sowohl materielle Interessen - wie in der Mikroökonomie den Akteuren als Handlungsprimat unterstellt wird - als auch Gemeinschaftsnormen - wie gemeinhin in der Soziologie argumentiert - führten zu dem spezifischen Verlauf der EU-Osterweiterung. Je nach Anreizintensität beider Einflussfaktoren variierte das Akteursverhalten. Durch die Erweiterung der Theorie des rhetorischen Handelns um das Konzept der Anomie wird nun nicht mehr nur die Phasenabfolge, sondern auch das konkrete Timing der Erweiterungspolitik verständlich. Diese Synthese aus Rationalismus und Konstruktivismus kann sich auch für andere Untersuchungsgegenstände als erklärungswirksames handlungstheoretisches Analyseinstrument erweisen, wenn Akteure ihre materiellen Interessen realisieren und im Lichte öffentlicher Aufmerksamkeit trotzdem ihr Image als verlässliches Gemeinschaftsmitglied nicht riskieren wollen. Da sich sowohl handelspolitische Partikularinteressen als auch Gemeinschafts normen als ausschlaggebend erwiesen, agierte die EU während ihrer historisch größten Erweiterung weder als ökonomischer Klub noch normengeleitete Wertegemeinschaft sondern als ,utilitaristische Wertegemeinschaft'.

\section{Bibliografia}

Brou D., Ruta M. (2004), A Positive Explanation of EU Enlargement, European University Institute Working Paper, Nr. 30.

Bulmer S., Paterson W. (1987), The Federal Public of Germany and the European Com munity, London.

Churchill W. (1946), Speech of 19 September 1946, available at: http://www.coe.int/T/E/Com/ about_coe/DiscoursChurchill.asp.

Durkheim É. (1993/1897), Der Selbstmord, übersetzt v. S. Herkommer, H. Herkommer, Frankfurt a.M.

Fierke K. M., Wiener A. (1999), Constructing Institutional Interests: EU and NATO enlar gement, RSC Working Paper, Nr. 14.

Garrett G. (1993), The Politics of Maastricht, „Economics and Politics“, Jg. 5, Nr. 2, S. 105-124.

Grabbe H., Hughes K. (1998), Enlarging the EU Eastwards, Royal Institute of International Affairs et al., London.

Götze C. (2003), Die Bedeutung von Staatsversagen und Anomie für zivilgesellschaftliche Organisationen. Beispiele aus Bosnien-Herzegowina und Albanien, in: Zivilgesellschaft-national und transnational, Hg. D. Gosewinkel, D. Rucht, W. Daele van den, J. Kocka, WZB-Jahrbuch, Berlin, S. 201-221. 
Hall P. A.; Taylor R. C. (1996), Political Science and the Three New Institutionalisms, „Political Studies“, Nr. 44, S. 936-57.

Kemmerling A. (2003), Die Osterweiterung der Europäischen Union: Ein polit-ökono mischer Erklärungsansatz, Beitrag zur Tagung der DVPW AK Integrationsforschung ,, Forschungslogik und Methoden der Internationalen Beziehungen und der Euro paforschung “, „Hofgeismar“, 2.-4.4.2003.

March J. G., Olsen J. P. (1989), Rediscovering Institutions. The Organizational Basis of Politics, New York.

Mehlhausen T. (2009a), Polens Rückkehr nach Europa. Warum sich die Europäische Union für ihre Osterweiterung entschied, Münster.

Mehlhausen T. (2009b), Der Streit um die doppelte Mehrheit. Deutsch-polnische Differenzen in der Europapolitik, in: Zwischen Skepsis und Vertrau en. Die deutsch-polnischen Beziehungen im Wandel, Hg. K. Mazurek, T. Mehlhausen, Jagiellonen-Universität, Krakau.

Milward A., Sorensen V. (1993), Interdependence or Integration? A National Choice, in: The Frontier of National Sovereignty: History and Theory, 1945-1992, Hg. A. Milward et al., London, S. $1-32$.

Moberg A. (2002), The Nice Treaty and Voting Rules in the Council, ,Journal of Com mon Market Studies“, Jg. 40, Nr. 2, S. 259-282.

Moravcsik A. (1998), The Choice for Europe. Social Purpose \& State Power from Messina to Maastricht, Ithaca-New York.

Moravcsik A., Vachudova M. A. (2002), Bargaining Among Unequals: Enlargement and the Future of European Integration, „European Union Studies Association Re view“, Jg. 15, Nr. 4, S. $1-4$.

Mulewicz J. (1992), Doświadczenia Polski w negocjacjach w sprawie Układu o stowarzyszeniu ze Wspólnotami Europejskimi, Warschau.

O'Brennan J. (2006), The Eastern Enlargement of the European Union, New York et al.

Papadimitriou D. (2002), Negotiating the New Europe. The European Union and Eastern Europe, Aldershot.

Plümper T., Schneider C. J. (2007), Discriminatory Membership and the Redistributi on of Enlargement Gains. A rational explanation of EU Membership Accession, „Journal of Conflict Resolution“, Jg. 51, Nr. 4, S. 568-587.

Pond E. (1993), Beyond the Wall: Germany's Road to Unification, Washington D.C.

Risse T., Börzel T. A. (2009), Venus Approaching Mars? The European Union as an Emerging Civilian Power, „Berliner Arbeitspapiere zur Europäischen Integration“, Nr. 11.

Saryusz-Wolski J. (1994), The Reintegration of the 'Old Continent': Avoiding the Costs of 'Half Europe', in: Economic and Political Integration in Europe: Internal Dynamics and Global Context, Hg. S. Bulmer, A. Scott, Oxford-Cambridge, S. 19-28.

Schimmelfennig F. (1999), The Double Puzzle of EU Enlargement. Liberal Norms, Rhetorical Action, and the Decision to Expand to the East, ARENA Working Paper, Nr. 15.

Schimmelfennig F. (2001), The Community Trap: Liberal Norms, Rhetorical Action, and the Eastern Enlargement of the European Union, „International Organization“, Jg. 55, Nr. 1, S. 47-80.

Schimmelfennig F. (2003), The EU, NATO and the Integration of Europe. Rules and Rhe toric, Cambridge.

Schimmelfennig F., Sedelmeier U. (Hg.) (2005a), The Politics of European Union enlarge ment. Theoretical approaches, London-New York.

Schimmelfennig F., Sedelmeier U. (Hg.) (2005b), The Europeanization of Central and Eastern Europe, Ithaca. 
Schneider C. J. (2009), Conflict, Negotiation, and European Union Enlargement, Cambridge.

Schuman R. (1950), Erklärung des französischen Außenministers, verfügbar auf: http://europa.eu/ abc/symbols/9-may/decl_en.htm.

Sedelmeier U. (2002), Sectoral dynamics of EU enlargement: advocacy, access and alliances in a composite policy, „Journal of European Public Policy“, Jg. 9, Nr. 4, S. 627-649.

Sedelmeier U. (2005), Constructing the Path to Eastern Enlargement. The uneven policy impact of EU identity, Manchester-New York.

Skalnes L. S. (2005), Geopolitics and the eastern enlargement of the European Union, in: The Politics of European Union enlargement. Theoretical approaches, Hg. F. Schimmelfennig, U. Sedelmeier, London-New York, S. 213-233.

Smith K. E. (2004), The Making of EU Foreign Policy. The Case of Eastern Enlargement, 2. Aufl., Palgrave Macmillan.

Torreblanca J. I. (2001), The Reuniting of Europe. Promises, negotiations and compromise, Aldershot.

Tsebelis G.e (1988), Nested Games. The Cohesion of French Politics, „British Journal of Political Science“, Jg. 18, Nr. 2, S. 145-170.

Vachudova M. A. (2005), Europe Undevided. Democracy, Leverage \& Integration After Communism, Oxford.

Vobruba G. (2004), Grenzen des Projekts Europa. Von der Expansionsdynamik zur abge stuften Integration, „OSTEUROPA“, Jg. 54, Nr. 5-6, S. 61-74.

Vobruba G. (2005), Die Dynamik Europas, Wiesbaden.

\section{Abstrakt \\ Wschodnie poszerzenie Unii Europejskiej. Anomiczne konsekwencje zjednoczenia Niemiec}

Zjednoczenie Niemiec przynioslo wielopłaszczyznowe konsekwencje dla procesu integracji europejskiej. Autor w artykule analizuje szeroką teoretyczną dyskusję, która toczyła się na ten temat w literaturze naukowej. Chodziło głównie o następstwa reunifikacji dwóch państw niemieckich dla Traktatu o Unii Europejskiej z Maastricht i poszerzenia Unii Europejskiej na wschód. 